\title{
Effects of short-to-long term enzyme replacement therapy (ERT) on skeletal muscle tissue in late onset Pompe disease (LOPD)
}

M. , R. Violano*, D. Ronchi†, S. Mondelloł, A. Nascimbeni§, I. Colombo*, G. Fagiolari*, A. Bordoni†, F. Fortunato†, V. Lucchini*, S. Simonaף, M. Filosto**, O. Musumeci††,

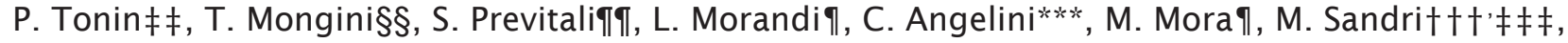
M. Sciacco*, A. Toscano††, G. P. Comi† and M. Moggio*

*Neuromuscular and Rare Diseases Unit, Department of Neuroscience, Fondazione IRCCS Ca' Granda, Ospedale Maggiore Policlinico, Milan, †Neurology Unit, Neuroscience Section, Department of Pathophysiology and Transplantation, Dino Ferrari Centre, IRCCS Foundation Ca' Granda Ospedale Maggiore Policlinico, University of Milan, Milan, $\neq$ Department of Biomedical and Dental Sciences and Morphofunctional Imaging, University of Messina, Messina, §Department of Neurosciences, University of Padova, Padova, IINeuromuscular Diseases and Neuroimmunology, Fondazione IRCCS Istituto Neurologico "Carlo Besta”, Milan, **Unit of Neurology, Center for Neuromuscular Diseases and Neuropathies, University Hospital "Spedali Civili”, Brescia, ††Department of Clinical and Experimental Medicine, Centro di Riferimento Regionale per le Malattie Neuromuscolari rare, University of Messina, Messina, \$Section of Clinical Neurology, Department of Neurological, Biomedical and Movement Sciences, University of Verona, Verona, §\$Department of Neurosciences “Rita Levi Montalcini”, University of Turin, Turin, IIIDivision of Neuroscience, Inspe, San Raffaele, Milan, ***Fondazione San Camillo Hospital IRCCS, Venice, $\pitchfork+D e p a r t m e n t$ of Biomedical Science, University of Padova and $\$+\$$ Dulbecco Telethon Institute at Venetian Institute of Molecular Medicine, Padova

M. Ripolone, R. Violano, D. Ronchi, S. Mondello, A. Nascimbeni, I. Colombo, G. Fagiolari, A. Bordoni, F. Fortunato, V. Lucchini, S. Simona, M. Filosto, O. Musumeci, P. Tonin, T. Mongini, S. Previtali, L. Morandi, C. Angelini, M. Mora, M. Sandri, M. Sciacco, A. Toscano, G. P. Comi and M. Moggio (2017)

Effects of short-to-long term enzyme replacement therapy (ERT) on skeletal muscle tissue in late onset Pompe disease (LOPD)

Aims: Pompe disease is an autosomal recessive lysoso- mal storage disorder resulting from deficiency of acid a- glucosidase (GAA) enzyme. Histopathological hallmarks in skeletal muscle tissue are fibre vacuolization and autophagy. Since 2006, enzyme replacement therapy (ERT) is the only approved treatment with human recombinant GAA alglucosidase alfa. We designed a study to examine ERT-related skeletal muscle changes in 18 modestly to moderately affected late onset Pompe disease (LOPD) patients along with the relationship

Correspondence: Maurizio Moggio, Neuromuscular and Rare Dis- eases Unit, Department of Neuroscience, Fondazione IRCCS Ca' Granda, Ospedale Maggiore Policlinico, via F. Sforza 35, 20122 Milano, Italy. Tel: +39 0255033851; Fax: $+3902550338027$.

E-mail: maurizio.moggio@unimi.it between morphological/biochemical changes and clini- cal outcomes. Treatment duration was short-tolong term. Methods: We examined muscle biopsies from 18 LOPD patients at both histopathological and biochemi- cal level. All patients underwent two muscle biopsies, before and after ERT administration respectively. The study is partially retrospective because the first biopsies were taken before the study was designed, whereas the second biopsy was always performed after at least 6 months of ERT administration. Results: After ERT, 15 out of 18 patients showed improved 6-min walking test (6MWT; $P=0.0007$ ) and most of them achieved respiratory stabilization. Pretreatment muscle biopsies disclosed marked histopathological variability, ranging from an almost normal pattern to a severe vacuolar 
myopathy. After treatment, we detected morphological improvement in 15 patients and worsening in three patients. Post-ERT GAA enzymatic activity was mildly increased compared with pretreatment levels in all patients. Protein levels of the mature enzyme increased in 14 of the 18 patients (mean increase $=+35 \%$;
$P<0.05)$. Additional studies demonstrated an improved autophagic flux after ERT in some patients. Conclusions: ERT positively modified skeletal muscle pathology as well as motor and respiratory outcomes in the majority of LOPD patients.

Keywords: acid alpha-glucosidase deficiency, autophagy, enzyme replacement therapy, Pompe disease

\section{Introduction}

Pompe disease (PD) (glycogen storage disease type II; OMIM 232300), is an autosomal recessive disorder caused by mutations in the acid a-glucosidase (GAA) gene on chromosome 17q25.2-q25.3 [1]. Affected indi- viduals accumulate excessive glycogen in the lysosomes and cytoplasm of several tissues. There are two major clinical manifestations of PD, namely infantile (IOPD) and late onset Pompe disease (LOPD) forms. LOPD is a slowly progressive myopathy involving axial, limb-gir- dle and respiratory muscles, often with a variable involvement of other organs such as the heart and cen- tral nervous system [2-4].

Since 2006, after the promising results from few prospective studies on animal models performed between the late nineties and the beginning of the years 2000 [5-7], the only approved treatment for PD is the enzyme replacement therapy (ERT) with recombi- nant human GAA (rhGAA; alglucosidase alfa, Myozyme ${ }^{\circledR}$; Sanofi Genzyme, Cambridge, MA, USA). In IOPD, early ERT administration reduces mortality and disability [8,9], whereas in LOPD patients it has shown variable improvement of motor performances and respi- ratory stabilization [10-12].

So far, few studies have examined ERT-related skele- tal muscle changes in PD patients [13-16]; however, the relationship between the morphological/biochemi- cal changes and the clinical outcomes are still unclear.

Autophagic mechanisms are implicated in multiple human diseases, including PD [17].

The autophagic pathway plays a crucial role in skeletal muscle homeostasis, providing a finely tuned system which mediates protein degradation and organelle removal [18]. Prior data suggest that autophagy improves myofiber survival in LOPD by facilitating GAA maturation, while its impairment contributes to muscle weakness and atrophy [19].
This retroscpective study analyses skeletal muscle tis- sues from 18 LOPD patients before and after ERT to survey how ERT can influence skeletal muscle pathobi- ology. All the data obtained were further correlated with patient clinical outcomes.

\section{Methods}

\section{Study design}

This study included 18 LOPD patients ( 8 males, 10 females) from seven Italian centres. All participants provided written informed consent, and the study pro- tocol and consent forms were approved by the ethics committee of each centre.

Clinical assessment was performed following established criteria $[3,20]$. PD diagnosis was confirmed at molecular level in all patients [21]. All patients exhibited axial, limb-girdle and/or respiratory muscles impairment at baseline (Tables 1, S1). ERT was admin- istered by intravenous infusions of alglucosidase alfa (Myozyme ${ }^{\circledR}$; Sanofi Genzyme) at a dose of $20 \mathrm{mg} / \mathrm{kg}$ every other week.

All patients underwent two muscle biopsies accord-ing to the following criteria: the first biopsy was per- formed for diagnostic purposes before the study was designed, namely 6 months - 3 years before starting ERT; the second one at least 6 months after ERT administration (Table 1) and 7-8 days after the latest ERT infusion. The first muscle biopsy was executed in one of the following muscles: quadriceps femoris, del- toid or biceps brachial. To make results comparable, we decided to perform the second one in the same, con-tralateral muscle in all patients.

\section{Clinical outcome measures}

From each patient, we obtained demographic and clini-cal data (Tables 1, S1). Patients were regularly 
Table 1. Summary of demographic, molecular and clinical data of the 18 patients with late onset Pompe disease

\begin{tabular}{|c|c|c|c|c|c|c|c|c|c|c|c|c|}
\hline \multirow[b]{2}{*}{ Patient } & \multirow[b]{2}{*}{ Gender } & \multirow[b]{2}{*}{$\begin{array}{l}\text { GAA mutations } \\
\text { (Allele } 1 \text {; Allele 2) }\end{array}$} & \multirow[b]{2}{*}{ Mutation effect } & \multirow[b]{2}{*}{$\begin{array}{l}\text { Age at onset } \\
\text { of symptoms } \\
\text { (years) }\end{array}$} & \multirow[b]{2}{*}{$\begin{array}{l}\text { Age at } \\
\text { diagnosis } \\
\text { (years) }\end{array}$} & \multirow[b]{2}{*}{$\begin{array}{l}\text { Age at ERT } \\
\text { beginning } \\
\text { (years) }\end{array}$} & \multirow[b]{2}{*}{$\begin{array}{l}\text { Mean duration } \\
\text { of disease at } \\
\text { ERT start } \\
\text { (years) }\end{array}$} & \multirow[b]{2}{*}{$\begin{array}{l}\text { Muscle } \\
\text { biopsy }\end{array}$} & \multicolumn{2}{|c|}{$\begin{array}{l}\text { Timing of the first } \\
\text { muscle biopsy }\end{array}$} & \multicolumn{2}{|c|}{$\begin{array}{l}\text { Timing of the } \\
\text { second muscle } \\
\text { biopsy }\end{array}$} \\
\hline & & & & & & & & & $\begin{array}{l}\text { Age } \\
\text { (years) }\end{array}$ & $\begin{array}{l}\text { Months } \\
\text { before } \\
\text { ERT start }\end{array}$ & $\begin{array}{l}\text { Age } \\
\text { (years) }\end{array}$ & $\begin{array}{l}\text { Months } \\
\text { after } \\
\text { ERT start }\end{array}$ \\
\hline 1 & M & c. $-32-13 \mathrm{~T}>\mathrm{G} ;$ c. $1927 \mathrm{G}>\mathrm{A}$ & p.Gly643Arg & 21 & 31 & 31 & 10 & Deltoid & 31 & 2 & 33 & 25 \\
\hline 2 & $\mathrm{~F}$ & c. $-32-13 \mathrm{~T}>\mathrm{G} ;$ c. $1076-1 \mathrm{G}>\mathrm{C}$ & $\begin{array}{l}\text { r.1076-79- } \\
1195+89 \text { ins }\end{array}$ & 42 & 51 & 52 & 10 & Quadriceps & 51 & 2 & 55 & 39 \\
\hline 3 & M & c. $-32-13 \mathrm{~T}>\mathrm{G} ;$ c. $1927 \mathrm{G}>\mathrm{A}$ & p.Gly643Arg & 30 & 66 & 67 & 37 & Quadriceps & 66 & 8 & 68 & 13 \\
\hline 4 & $\mathrm{~F}$ & c. $-32-13 \mathrm{~T}>\mathrm{G}$; c.525delT & p.Glu 176 Argfs* 45 & 40 & 51 & 51 & 11 & Quadriceps & 51 & 5 & 53 & 16 \\
\hline 5 & $\mathrm{M}$ & c. $-32-13 \mathrm{~T}>\mathrm{G} ;$ c. $525 \mathrm{delT}$ & p.Glu176Argfs $* 45$ & 65 & 72 & 72 & 7 & Biceps & 72 & 3 & 72 & 10 \\
\hline 6 & $\mathrm{M}$ & c. $-32-13 \mathrm{~T}>\mathrm{G} ;$ c $.1655 \mathrm{~T}>\mathrm{C}$ & p.Leu552Pro & 25 & 56 & 57 & 32 & Quadriceps & 56 & 13 & 57 & 7 \\
\hline 7 & $\mathrm{~F}$ & c. $-32-13 \mathrm{~T}>\mathrm{G} ;$ c. $2237 \mathrm{G}>\mathrm{A}$ & p. $\operatorname{Trp} 746^{*}$ & 37 & 38 & 40 & 3 & Quadriceps & 41 & 14 & 41 & 15 \\
\hline 8 & M & c. $-32-13 \mathrm{~T}>\mathrm{G} ; \mathrm{c} .525 \mathrm{delT}$ & p.Glu176Argfs ${ }^{*} 45$ & 16 & 36 & 36 & 20 & Quadriceps & 36 & 8 & 37 & 8 \\
\hline 9 & $\mathrm{M}$ & c. $-32-13 \mathrm{~T}>\mathrm{G} ;$ c $.525 \mathrm{delT}$ & p.Glu176Argfs* 45 & 46 & 48 & 48 & 2 & Quadriceps & 48 & 5 & 49 & 8 \\
\hline 10 & M & c. $-32-13 \mathrm{~T}>\mathrm{G}$; c. $784 \mathrm{G}>\mathrm{A}$ & p.Glu262Lys & 50 & 52 & 53 & 3 & Quadriceps & 52 & 17 & 54 & 8 \\
\hline 11 & $\mathrm{~F}$ & c.-32-13T>G; c.525delT & p.Glu176Argfs*45 & 48 & 54 & 54 & 6 & Quadriceps & 54 & 1 & 55 & 12 \\
\hline 12 & $\mathrm{~F}$ & c. $-32-13 \mathrm{~T}>\mathrm{G} ;$ c. $1561 \mathrm{G}>\mathrm{A}$ & p.Glu521Lys & 48 & 58 & 59 & 11 & Quadriceps & 58 & 7 & 59 & 7 \\
\hline 13 & $\mathrm{M}$ & c. $-32-13 \mathrm{~T}>\mathrm{G} ;$ c. $525 \mathrm{delT}$ & p.Glu176Argfs*45 & 34 & 45 & 45 & 11 & Quadriceps & 45 & 7 & 46 & 12 \\
\hline 14 & $\mathrm{~F}$ & c. $-32-13 \mathrm{~T}>\mathrm{G} ;$ c. $1927 \mathrm{G}>\mathrm{A}$ & p.Gly643Arg & 15 & 16 & 16 & 1 & Biceps & 16 & 6 & 17 & 7 \\
\hline 15 & $\mathrm{~F}$ & c. $-32-13 \mathrm{~T}>\mathrm{G} ;$ c. $2237 \mathrm{G}>\mathrm{A}$ & p.Trp $746^{*}$ & 39 & 40 & 41 & 2 & Deltoid & 40 & 6 & 41 & 8 \\
\hline 16 & $\mathrm{~F}$ & c. $-32-13 \mathrm{~T}>\mathrm{G} ;$ c. $1465 \mathrm{G}>\mathrm{A}$ & p.Asp489Asn & 37 & 49 & 50 & 13 & Quadriceps & 49 & 14 & 54 & 46 \\
\hline 17 & $\mathrm{~F}$ & c. $-32-13 \mathrm{~T}>\mathrm{G} ;$ c. $2331+1$ & Loss of transcript & 30 & 39 & 41 & 11 & Quadriceps & 39 & 22 & 45 & 57 \\
\hline 18 & $\mathrm{~F}$ & c. $-32-13 \mathrm{~T}>\mathrm{G} ;$ c. $1465 \mathrm{G}>\mathrm{A}$ & p.Asp489Asn & 50 & 53 & 56 & 6 & Biceps & 53 & 31 & 57 & 8 \\
\hline Mean & & & & 36.8 & 46.9 & 47.6 & 10.9 & & 47.1 & 8.9 & 49.1 & 17.0 \\
\hline $\begin{array}{l}\text { Mean } \\
(\mathrm{SD})\end{array}$ & & & & 13.2 & 13.1 & 13.2 & 10.2 & & 13.0 & 8.1 & 13.1 & 14.9 \\
\hline
\end{tabular}

GAA, acid alpha-glucosidase; ERT, enzyme replacement therapy. 
monitored and evaluated following previously reported protocols [20,22]. Forced vital capacity (FVC) was spiro- metrically assessed in the sitting position, and expressed as a percentage of the predicted value. Motor function was assessed by the 6-min walking test (6MWT).

\section{Morphological study}

All samples were analysed by operators from two referring centres: Foundation IRCCS $\mathrm{Ca}^{0}$ Granda University of Milan and Institute C. Besta, Milan. Samples were both fixed in $2.5 \%$ glutaraldehyde $(\mathrm{pH}$ 7.4 ), and frozen in liq- uid nitrogen, and processed using standard techniques [23]. Three different blinded operators evaluated each biopsy following the protocol by Peverelli et al. [24]. For each biopsy, four digitized non-overlapping consecutive pictures were taken of four randomly selected areas of the same cryostatic section stained with GT, AP and Per- iodic Acid-Schiff (PAS). Pictures were taken at 920 magnification using a camera-equipped Leica DC200 optic microscope (Leica Microsystems Srl, Milano, Italy) and then examined using image analyzer software IM50 (Leica Microsystems Srl, Milano, Italy). In order to iden- tify and assess the effect of ERT on vacuolated fibres, We established a rigorous protocol for classification and developed a scoring system to define treatment response. In detail, for each biopsy, total number of fibres, percent- age of vacuolated fibres and percent coefficient of varia- tion (CV) were calculated. By GT and AP staining, we created a four-point graded scale to classify vacuolated fibres according to vacuole number and size (from mild to very severe vacuolation) (Figure S1A). The percentage of different types of vacuolated fibres was assessed in pre- and post-ERT biopsies and a relative change $\geq 20 \%$ ( $>3$ standard deviation of the $\mathrm{CV}$ ) served as the standard cri- terion to determine relevant improvement or worsening, using similar criteria described for the evaluation of dif- ferent biomarkers. [25,26]. Furthermore, a vacuolation weighted delta (VWD) score, scoring the changes across the four-point graded scale between pre- and post-ERT biopsies, was created to determine the 'Overall Vacuolation Response' to the ERT and used for the analysis (Fig- ure S1B). For PAS staining intensity, we used a scale ranging from + (mild) to +++ (severe) (Table S2) [27] in both cryostatic and semithin sections. We also analysed the following histological features: fibre size variability, fibre atrophy and hypertrophy, the presence of angu- lated fibres and fibre splitting, increased connective tissue, fibre necrosis, the presence of internal nuclei (Table S3) as previously reported [24,27].

\section{Morphometric analysis}

For morphometric analysis, ATPase fibre-type staining at $\mathrm{pH} 4.3,4.6$ and 9.4 was performed on Optimum Cutting Temperature (OCT)-embedded frozen tissue sec- tions following standardized protocols. Using four con- secutive microphotographs of sections stained for ATPase at $\mathrm{pH}$ 9.4, we calculated the vacuolated fibre percentage for each fibre typology (Table S4).

Determination of acid alpha-glucosidase activity in muscle

For biochemical analysis, muscle specimens were immediately frozen in liquid nitrogen and stored at

$-80^{\circ} \mathrm{C}$. Acid alpha-glucosidase deficiency was measured before and after ERT by fluorometric determina- tion of enzyme activity [28].

\section{Protein studies}

Protein samples obtained from fresh-frozen muscle biop- sies were immunoblotted as previously described [29], Glyceraldehyde 3-phosphate dehydrogenase (GAPDH) was used as a loading control. We performed densitomet- ric quantification from multiple gels for each experiment using ImageJ software (US National Institutes of Health). Antibodies used for western blot analysis included pri- mary antibody to microtubule-associated protein 1 light chain 3 b (LC3II) (Sigma-Aldrich, S.r.l. Milan, Italy), antibody to SQSTM1 (GSQSTM1-C) (Progen Biotechnik GmbH, Heidelberg, Germany), antibody to GAA (Sanofi Genzyme, Cambridge, MA, USA), antibody to GAPDH (Abcam, Cambridge, United Kingdom), and Horseradish Peroxidase (HRP)labelled secondary anti-mouse, anti- rabbit (GE Healthcare, Milan, Italy), and anti-guinea pig antibodies (Sigma-Aldrich).

\section{Statistical analysis}

Statistical analyses were performed using Stata software (Version 13.0; Stata Corp LLC, Texas, USA) and SAS (version 9.0; SAS Institute Inc., Cary, NC, USA).

Data were assessed for equality of variance and distri-bution. Descriptive statistics with means and medians, 
as appropriate, and proportions were used to describe continuous and categorical variables. Paired $t$-tests were used to assess changes in Body Mass Index (BMI), FVC and 6MWT. For statistical comparison of non-normally distributed continuous variables, we used the MannWhitney $U$ test in case of two groups and the KruskalWallis test in case of three or more groups. Correlation analyses were performed by means of the nonparamet- ric Spearman rank correlation test. The association between categorical variables was evaluated using the Fisher's exact test. All statistical tests were two-tailed and a $P$ value $<0.05$ was considered significant.

\section{Results}

Description of population and clinical outcomes before and after ERT

In our cohort $(n=18), 55.6 \%$ (10) of the subjects were women, the average patient age at onset of symptoms was $37.39 \mathrm{~T} 13.1$ years, while the average patient age at diagnosis was $47.5 \mathrm{~T} 13$ years, with a median diag- nostic delay of 9 years (range $=1-36)$. The mean BMI was 23.13 T 3.75.

Seventeen patients were fully ambulant and one used a walker before ERT. The median walking distance was

$393.9 \mathrm{~m}$ (T127.0) and the mean FVC in the sitting position was $81.7 \%$ (T23.2). Two (11\%) patients required ventilatory support for less than $12 \mathrm{~h}$ a day before starting ERT.

ERT treatment was initiated in all patients at variable ages after symptoms onset (range $=1-37$ years) and about 1 year (0-3) after diagnosis.

After ERT, 15 patients improved in 6MWT (mean distance walked $448.6 \mathrm{~T} 127.1 \mathrm{~m}, P=$ $0.0007)$ while no significant changes were observed in FVC (mean post-treatment 80.4T22.2\%, $P=$ 0.79). Six patients (in addition to the initial two) needed the respiratory sup- port (four for $<12$ h/day, two for longer).

BMI did not change after treatment (mean $=23.19$ T 3.76, $P=0.71$; Table $1, \quad \mathrm{~S} 1)$.

Morphological evaluation of muscle biopsies before and after ERT

Morphological findings resulted from the observations of three different blinded operators from the two refer- ring centres as described (see Methods).
Pretreatment muscle biopsies showed marked histopathological variability, ranging from an almost normal morphology to a severe vacuolar myopathy. The most common abnormalities included muscle fibres with PAS-positive vacuoles of varying number and size (Figure 1) and a glycogen storage. The comparison of pre- and post-ERT vacuolated fibres of the 18 patients showed morphological improvement in 15 patients, and worsening in 3 patients according to the VWD score (Figures 2, S1B). In particular, after ERT, in 15 patients, we observed a significant reduction in the pro- portion of vacuolated fibres of grade + (mild) and ++ (moderate) compared to fibres of grade +++ (severe) and ++++ (very severe) $(67 \%$ vs. $39 \%, P=0.0275)$.

The 15 muscle biopsies showing vacuolated fibre reduction also showed a relevant glycogen reduction, whereas the three worsened biopsies showed glycogen increase. Notably, we observed that small PAS-positive accumulations disappeared in all post-treatment biop- sies, whereas large PASpositive collections were unchanged or increased. These data were particularly evident at PAS staining of frozen and semithin sections evaluation (Figure 1, Table S2).

The histochemical acid phosphatase activity was abnormal in all patients and paralleled both number and size of vacuoles along with a variable intracytoplasmic activity.

Pretreatment biopsies showed internal nuclei in 17 patients and large fibre size variability in most sam- ples, with coexistence of atrophic and hypertrophic fibres. Scattered angulated fibres were seen in muscle biopsies from 16 patients. These latter aspects remained basically unchanged in posttreatment biop- sies (Table S3).

\section{Morphometric analysis}

In the first biopsy, 15 patients showed nonselective involvement of muscle fibre types; a slight prevalence of type 1 vacuolated fibres was present in seven patients, whereas type 2 vacuolated fibres were some- what predominant in two patients. In addition, patients 7 and 17 showed selective vacuolization in type 1 mus- cle fibres, while patient 14 revealed only vacuolated fibres of type 2 . These patterns remained nearly unchanged in posttreatment biopsies, except for patient 16, where the muscle fibre percentages reversed (Table S4). 

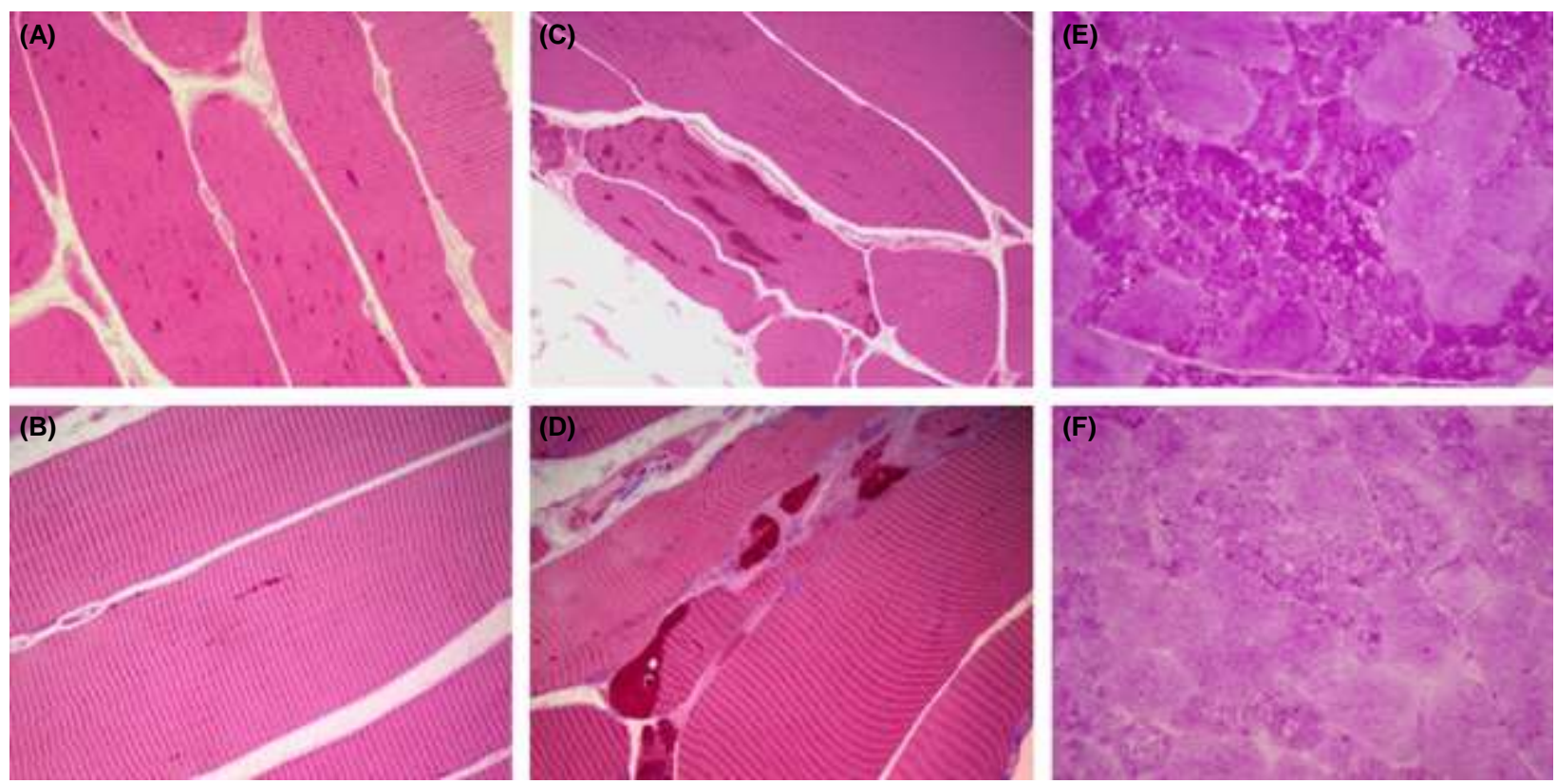

Figure 1. PAS staining in muscle biopsies before and after ERT. Representative pictures of muscle PAS staining (example of pt number 14). (A-D) PAS staining on semithin sections; (E,F) PAS staining on frozen sections. Upper panel: pretreatment muscle biopsy, lower panel: post-treatment muscle biopsy. Small PAS-positive accumulations (A) disappear in post-treatment biopsies (B) whereas the large PAS-positive collections (C) are unchanged or increased (D). PAS staining shows an important glycogen accumulation in pretreatment muscle tissue (E), which is reduced after therapy (F). Original magnification: A,C: 9 400; B,D: 9 1000; E,F: 9250

Molecular characterization, muscle acid alpha- glucosidase activity and protein levels

All patients harboured the heterozygous IVS1-13T $>$ G mutation, affecting exon 2 within $G A A$ transcript. The second allele carried truncating and out-of-frame muta- tions (10/18) or known missense variants (8/18). Six patients $(33 \%)$ harboured the c.525delT microdeletion, the second most common molecular defect. Before ERT, mean residual activity of acid alpha-glucosidase in muscles was $8.45 \mathrm{~T} \quad 3.22$ $\mathrm{pmol} / \mathrm{min} / \mathrm{mg}$ protein (refer- ence value: $113 \mathrm{~T} 41$ $\mathrm{pmol} / \mathrm{min} / \mathrm{mg}$ protein) (Fig- ure 3B). The lowest residual activity levels were observed in patients harbouring a non-sense mutation or variant resulting in amino acid change near the enzyme's catalytic site. The mean residual activity sig- nificantly improved after ERT $(11.31 \mathrm{~T} 2.87 \mathrm{pmol} / \mathrm{min} / \mathrm{mg}, P<0.05)$ (Figure 3B). The increased enzyme activity greatly varied, ranging from $+7.7 \%$ in patient

6 to $+202.6 \%$ in patient 16 . Patients 10 and 12 showed the highest GAA activity levels both before and afterERT (Figure 3A).

We also evaluated GAA protein level by western blot analysis. Muscle specimens displayed a maximum of three major bands corresponding to preprotein $(110 \mathrm{kDa})$ and mature forms of the enzyme (76 and $70 \mathrm{kDa}$ ). The $110-\mathrm{kDa}$ band was detected in patients harbouring missense mutations, particularly in patients with defects known to impair GAA enzyme physiologi- cal maturation (patient 12 with pGlu521Lys, and patient 6 with pLeu552Pro).

After GAPDH correction, densitometric analysis revealed increased ( $>35 \%$ ) GAA mature forms in the second biopsies of 14 patients, with five of them show- ing a doubled GAA signal intensity post-ERT. Protein levels decreased in the remaining four subjects (Fig- ure 3C). The ratio between mature and preprotein forms (where observed) was unchanged before and after ERT in six subjects and modestly increased after ERT in the remaining patients. Overall, after ERT, we observed a positive correlation between mature protein levels and residual alpha glucosidase activity in most patients (Figure 3D).

ERT treatment preserved this correlation and resulted in a modest increase of active acid alphaglucosidase enzyme. There was a positive correlation at both first $(R=0.74, \quad P=0.0005) \quad$ and second $\quad(R=0.55$,

$P=0.022$ ) biopsy (Figure 3D). 
(A)

Vacuolation weighted delta (VWD) score of our population

\begin{tabular}{|c|c|c|c|c|c|c|c|c|c|c|c|c|c|c|}
\hline \multirow{3}{*}{ Sobject in } & \multicolumn{12}{|c|}{ Percentage ofDifferent type of Vxcuobated Fibers in Pre and Post ERT Biopsies and Relatire Scores } & \multirow[b]{3}{*}{ Sen Senr } & \multirow{3}{*}{$\begin{array}{c}\text { Orerall } \\
\text { Vacuolatine } \\
\text { Renpesu }\end{array}$} \\
\hline & \multicolumn{3}{|c|}{ Miad racuelatios } & \multicolumn{3}{|c|}{ Mederate racselation } & \multicolumn{3}{|c|}{ Senere racuabetaes } & \multicolumn{3}{|c|}{ Tery mere mactedatios } & & \\
\hline & Prother & Pinstent & Senre 1 & Pentent & Pvis-txt & Searn II & Proxat & Phistent & seore $\mathrm{m}$ & Protert & Pwitext & $\operatorname{sen} v 17$ & & \\
\hline 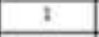 & 25 & Q7! & 2 & $2: 24$ & ? & 9 & 157 & 2 & 8 & 257 & 0 & 2 & 1 & ingeriat \\
\hline 2 & 419 & 331 & 8 & $3 x$ & 8 & 5 & 208 & 2 & $\theta$ & 297 & 0 & 8 & 1 & erpest \\
\hline 3 & 4 & 29 & 0 & 21 & iti & 2 & 72 & 0 & 9 & 75 & 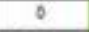 & 2 & 1 & esprotsi: \\
\hline 4 & 12 & 14 & 1 & 118 & 88 & 2 & Q3. & 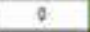 & 9 & 11.4 & 21. & 2 & $:$ & inpoeged. \\
\hline 2 & 300 & 09 & 0 & $0 \pi$ & 2 & 9 & 0 & 0 & 22 & 0 & 0 & 0.9 & 3 & inpond \\
\hline 5 & 45. & 105 & 2. & 21 & on & 8 & 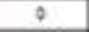 & 0 & 05 & 0 & 0 & $0:$ & $:$ & impoenst: \\
\hline 7 & 17 & 93 & 025 & 41 & 22 & t & 238 & 10 & 2 & 0 & 2 & 62 & $\therefore$ & mprost \\
\hline 1 & 1136 & $12: 2$ & 02 & 293 & 221 & 9 & Q75 & 0 & 9 & 9 & 0 & 0.2 & $:$ & mpored \\
\hline 7 & 11 & 211 & 025 & 12 & 2 & $\theta$ & 8 & 8 & 25 & 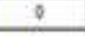 & 2 & 0.2 & 3 & mperent \\
\hline 10 & 47 & 1.7 & 0 & 0 & $\theta$ & 02 & 0 & 0 & 09 & 0 & $\theta$ & 0.9 & 23 & inporines \\
\hline 11 & 5.1 & 33 & 2 & 328 & 165 & 2 & 102 & 175 & 2 & 2 & 11 & 2 & 2 & inperent: \\
\hline 21 & 694 & 178 & 0. & $43 t$ & 3.54 & 2 & 258 & 485 & 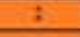 & 411 & $2 \%$ & 2 & 1 & inponed \\
\hline HI & 17 & 22 & 1 & 135 & 14 & 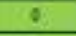 & 17 & 43 & 3 & 4.1 & 0 & 3 & 4 & intereond \\
\hline 14 & $12 t$ & 14 & 32 & 2.84 & 131 & 8 & 248 & IN & 9 & 49 & 27 & 22 & 4 & mprost \\
\hline H & 1204 & $29 !$ & 0 & 179 & 333 & Q.5 & $\phi$ & 08 & 2 & 9 & 0 & 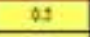 & + & mporent \\
\hline 16 & $\pi$ & 159 & 1 & 165 & 201 & 19 & 9 & 912 & $z$ & 9 & $\theta$ & 62 & 4 & vernet \\
\hline It & 48 & its & 22 & 179 & 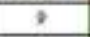 & 9 & 9.79 & 47 & 2 & 0 & 12 & 25 & 1 & nunated \\
\hline in & 196. & c4 & 1. & 0.93 & Q.4 & 2 & 9 & 04 & 2 & 9 & $2 .+$ & 21 & is: & Wantunt: \\
\hline
\end{tabular}

(B)

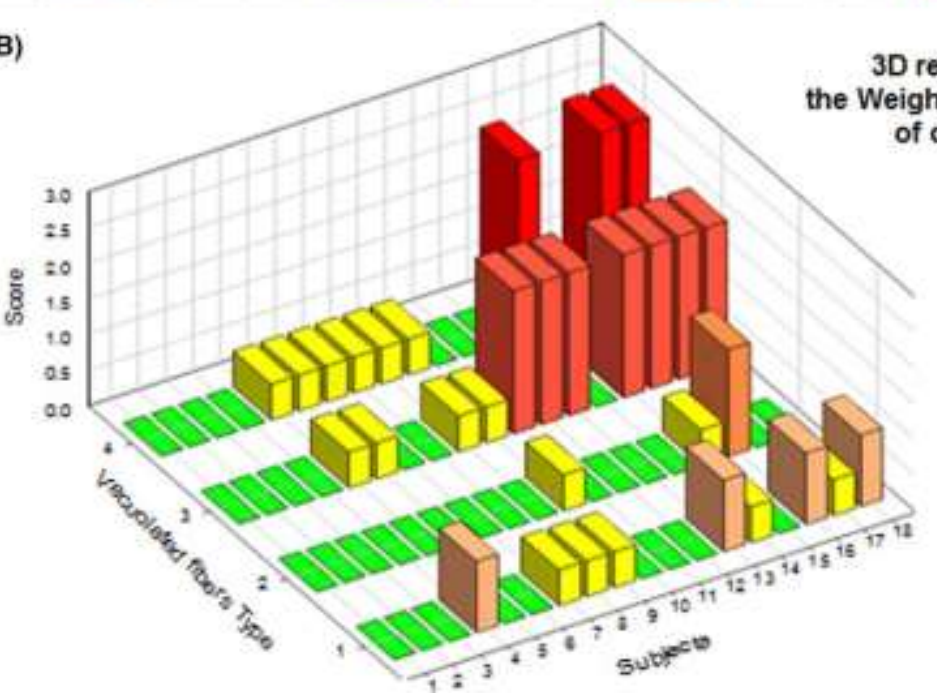

Figure 2. Morphological evaluation of muscle biopsies before and after ERT. (A) Vacuolation weighted delta (VWD) score of our population. (B) Three-dimensional representation of the VWD score of our population.

Analysis of the autophagic pathways

To determine whether ERT affected autophagy, we monitored the LC3 lipidation level as readout of autophagosome and the expression of the autophagy substrate, p62/SQSTM1, as an index of lysosomaldependent clearance.

LC3II analyses revealed heterogeneity. Indeed, ERT determined LC3II increase in five patients, no changes in three patients and a reduction in five patients (Figure 4A).
Post-treatment biopsies also showed decreased p62/ SQSTM1 protein in eight patients and an accumulation in five patients (Figure 4B). Three of these latter patients (patients 8, 13, and 16) also displayed LC3II accumulation.

\section{Discussion}

ERT with rhGAA is currently the only therapy approved for PD. The beneficial effects in improving survival, maintaining motor functions over time as well 


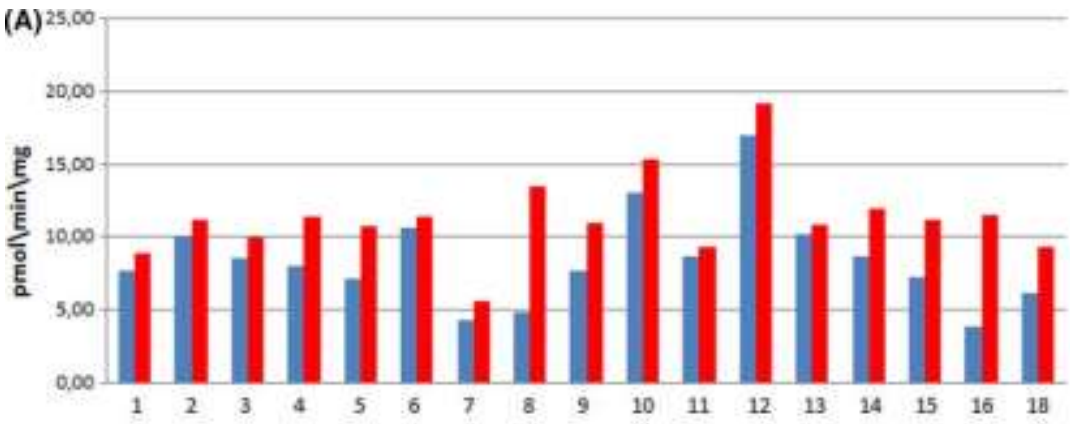

(C)

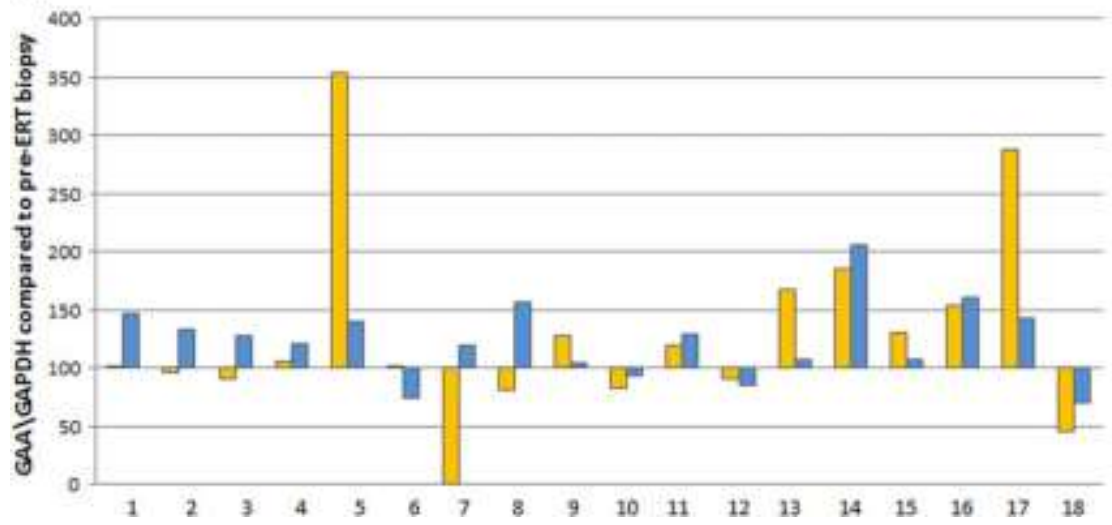

(B)

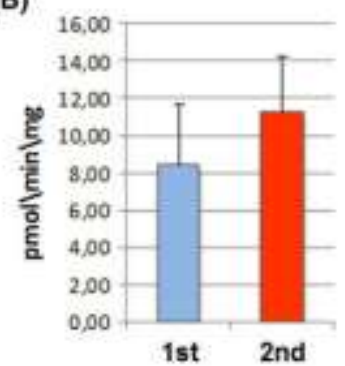

(D)

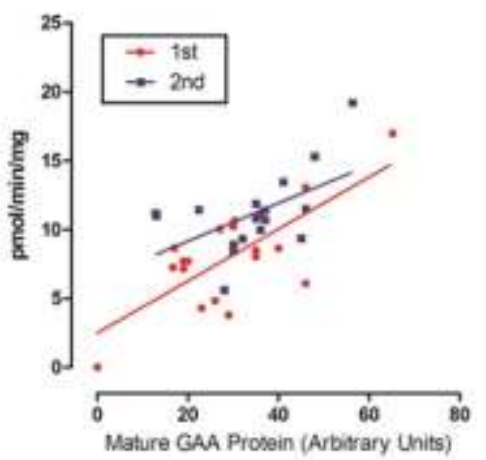

Figure 3. Biochemical analysis in muscle biopsies before and after ERT. (A) Quantification of residual acid alpha-glucosidase activity in late onset Pompe disease patients before (blue) and after (red) ERT administration. (B) Mean values of the two series $(P<0.01)$ Values are expressed as pmoles product formed per min and per mg protein. (C) Densitometric analysis of GAA levels from different blots.

Expression of immature (yellow) and mature (blue) GAA forms (110 and $95 \mathrm{kDa}$ : inactive precursor and immature forms,

respectively; 76 and $70 \mathrm{kDa}$ : mature lysosomal forms) in patients compared to untreated biopsies. (D) Correlation between mature GAA protein levels ( $x$-axis, arbitrary units from densitometric analysis) and residual acid alpha-glucosidase activity ( $y$-axis, values expressed as pmol/min/ $\mathrm{mg}$ ) in muscle biopsies before (red circles) and after (blue squares) ERT administration.

as preventing severe deterioration or stabilize respira- tory parameters have been demonstrated in LOPD patients [3,10-12].

We examined skeletal muscle biopsies from 18 LOPD patients before and after ERT treatment lasting from 7 to 57 months. The second biopsy was performed after at least 6 months of ERT administration (Table 1) and 7-8 days after the latest ERT infusion. Our population is heterogeneous because patients were recruited retro- spectively and therefore underwent the first muscle biopsy, taken for diagnostic purposes, with variable timing before the study was designed. This aspect, which would represent a bias in case of a purely exper- imental study, turned into a useful instrument in our study because it gave us the opportunity to examine ERT effects also in subjects (and skeletal muscles) who were treated for a long time and/or who started ERT several months/years after the diagnosis was made.
Our patients showed significant post-treatment improvement at 6MWT. The majority of them obtained respiratory stabilization, although six patients, in addi- tion to the initial two, required respiratory support (four for $<12 \mathrm{~h} /$ day, two for a longer period) (Table S1).

In pretreatment muscle biopsies, the most common histological abnormalities were vacuolated fibres, accu- mulation of PAS-positive material (glycogen), and increased acid phosphatase activity. Comparison of glycogen content and vacuole number between pre- and post-treatment biopsies revealed improvement in 15 patients, and worsening in three patients.

The best outcome in second biopsies was observed when the architecture of the skeletal muscle fibres was not so severely impaired in pretreatment biopsy, namely when the vacuoles were of a minor degree. Also, we noticed the disappearance of small PAS- 

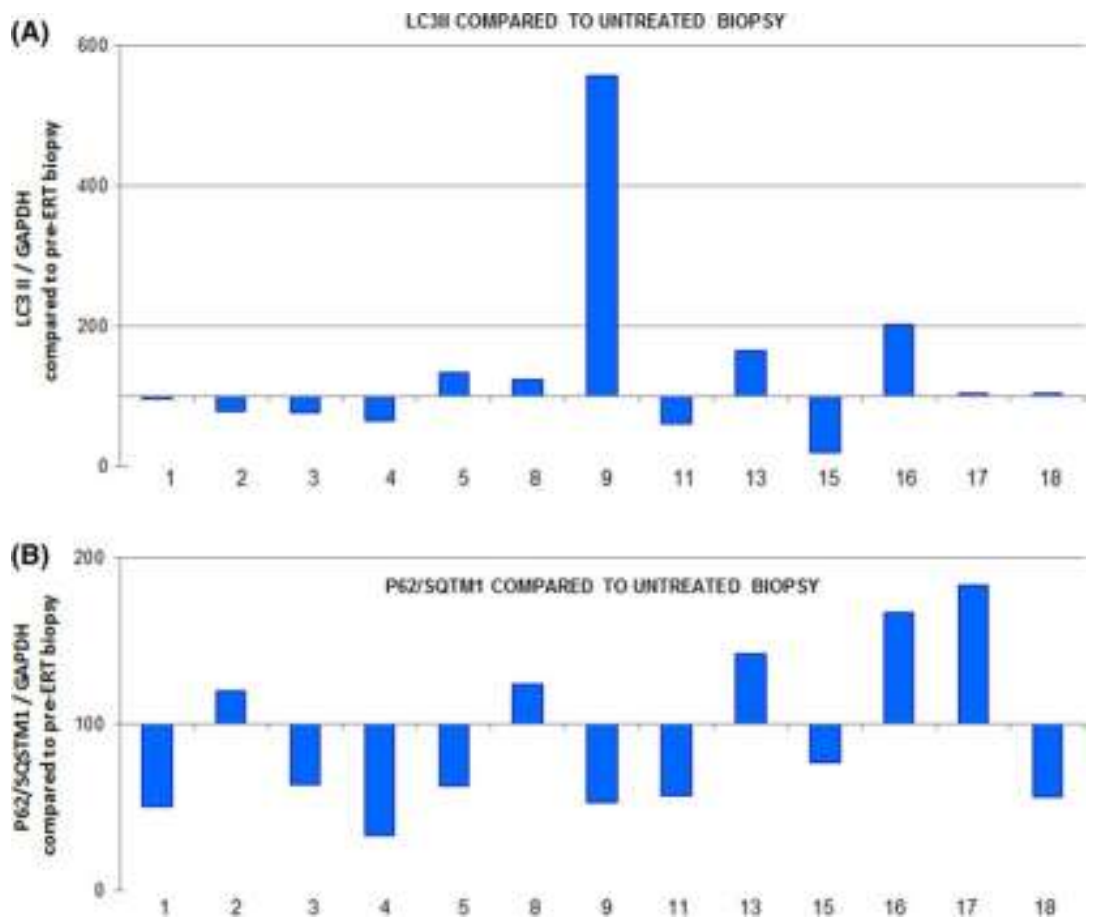

Figure 4. Densitometric analysis of LC3II, and p62/SQSTM1. Densitometric analysis of (A) LC3II, and (B) p62/SQSTM1 normalized to glyceraldehyde 3-phosphate dehydrogenase (GAPDH). Different blots evaluated protein expression in 13 patients compared to untreated biopsies.

positive accumulations in post-treated biopsies, whereas larger PAS-positive collections were almost unchanged (Figure 1).

Importantly, we demonstrated a differential effect of the ERT therapy on the vacuolated fibres. Specifically, a significantly higher proportion of grade + (mild) and ++ (moderate) vacuolated fibres improved as compared to grade +++ (severe) and ++++ (very severe) vacuolated fibres $(67 \%$ vs. $39 \%, P$ $=0.0275)$. These findings suggest that the ERT therapy produces a greater beneficial effect on the less affected fibres, thus supporting the idea that the treatment is most effective when initiated in early stages of PD, before severe and very severe vacuolated fibres appear and become too widespread. These conclu- sions resulted from the observations of three different blinded operators from the two referring centres.

These data are in agreement with those of van der Ploeg et al.[14], who show a beneficial ERT effect on small glycogen accumulations, whereas the larger glycogen pools, which are the hallmark of the most damaged fibres, remained refractory to treatment. These morphological considerations may help clinicians assess the most appropriate timing to start treatment.
Moreover, lack of progression of the disease might be an important factor as well as the improvement.

Studies in knockout mice show that autophagic vacuole accumulation in skeletal muscle is mainly restricted to type 2 fibres, and that type 1 fibres respond better to ERT than type 2 fibres [7,30], most likely because muscles with predominantly type 1 (slow-twitch) fibres, namely soleus and cardiomyocytes, have a higher capillary density [31]. Conversely, autop- hagic accumulation in humans involves both type 1 and type 2 muscle fibres [32,33].

Among our 18 patients, before ERT, nine muscle samples showed prevalent involvement of type 1 muscle fibres, whereas three samples mainly showed vacuo- lation in type 2 fibres. These features were unchanged in post-treatment biopsies. The other six specimens showed a non-selective involvement of muscle fibre types. We concluded that the vacuolated fibre typology does not correlate with disease severity nor does it pre- dict morphologic evolution, at least in the skeletal mus- cles we examined. Indeed, vacuole fibre type localization changed in a few treated subjects indepen- dently on morphological outcomes (Table S4). 
Scattered angulated fibres were seen in muscle biopsies from 16 patients and we found no modifications in post- treatment biopsies (Table S3). The neurogenic origin of the scattered angulated fibres we found cannot be excluded and Electromyography (EMG) findings (coexis- tence of myopathic and mild to moderate neurogenic signs) are compatible with this hypothesis in some of our patients. Indeed, as already suggested by other authors [34], glycogen storage in muscleinnervating structures may account for the presence of neurogenic signs in muscle biopsies.

Post-treatment muscle biopsies worsened in patients 16,17 and 18 . In patients 16 and 17 , very long time had passed between disease onset and ERT start, whereas the delay was shorter in patient 18. The diag- nostic delay may have negatively influenced the ability of ERT to restore or limit muscle alterations, especially when large vacuoles were present. Also, patient 18 underwent second muscle biopsy after 8 months of ERT, whereas pts 16 and 17 after 46 and 57 months respectively. In the latter, we can also postulate that the bioptic worsening has followed an initial improve- ment. These observations indicate that bioptic outcome and ERT duration do not easily correlate. Despite the histological worsening, all these patients improved at 6MWT with minimal changes in FVC. Thus, we may speculate that the contractile force of nondegenerated muscle fibres improves, despite worsening of some mor- phological parameters in selected muscle areas.

The opportunity to study muscle tissue before and after ERT allowed us to also perform biochemical anal- ysis in 17 patients. All of them showed moderately increased GAA enzymatic activity in post-treatment skeletal muscles, in accordance with a slightly increased level $(>35 \%)$ of GAA mature forms in 14 patients. These differences were not related to specific patient genotypes. ERT is intended to increase the residual enzyme activity enough to induce measurable clinical improvement. While IOPD is always associated with complete or nearlycomplete deficiency of GAA enzyme activity $(<1 \%$ of control values), LOPD is asso- ciated with reduced enzyme activity ranging from $1 \%$ to $30 \%$ of normal $[35,36]$. Notably, patients carrying the same enzymatic activity may present different clini- cal patterns. We may postulate the existence of a specific patient-related threshold of enzymatic activity below which a presymptomatic/oligosymptomatic patient becomes overtly symptomatic [37]. So, subjects with residual activity just below this threshold may show clinical improvement with only a slight increase in enzymatic activity.

The autophagic pathway plays a crucial role in skeletal muscle homoeostasis, providing a finely tuned system to mediate protein degradation and organelle removal [18]. In PD, autophagy impairment con- tributes to muscle pathology in the animal model (GAA-ko) [17] and in human muscles [19]. To deter- mine whether autophagy was influenced by ERT, we monitored LC3 lipidation levels as a reflection of autophagosomes, and the expression of the autophagy substrate p62/SQSTM1 as an index of lysosomal-depen- dent clearance.

Since PD is characterized by autophagosome buildup, an increase of LC3II may reflect impaired autophagosome degradation as well as an induction of autophagosome biogenesis. A reduction of the LC3II band after ERT, could indicate increased autophagosome clearance. LC3II analysis revealed a certain heterogeneity, with ERT leading to increased LC3II in five patients, no change in three patients, and reduced LC3II in five patients.

To better understand whether autophagy was impaired or activated, we monitored expression of the autophagy substrate p62/SQSTM1. Interestingly, ERT led to decreased p62/SQSTM1 protein in eight patients and to p62/SQSTM1 accumulation in five patients. Three of the five patients with p62/SQSTM1 accumula- tion (patients 8,13 and 16) also displayed LC3II accu- mulation, suggesting that autophagy was not reactivated by ERT. The decreased p62 in eight patients suggested autophagy system improvement after ERT. These findings are mostly in agreement with previous studies in animal models that have shown a clear cor- relation between autophagosome accumulation and ERT ineffectiveness. Importantly, ERT requires a func- tional cell-trafficking system to allow delivery of recom- binant enzyme to the lysosomes.

The comparison between untreated and treated biop- sies in this group of patients represents an important contribution to the understanding the role of autophagy and the effects of ERT on muscle tissue. However, our results were discordant in some patients; for example, the post-treatment biopsy from patient 8 showed wors- ened features compared to the pre-treatment biopsy. In detail, western blot analysis showed an accumulation of LC3II and P62 in the post-treatment biopsy. It is 
difficult to understand how these negative features cor- relate with co-existing increased GAA matureform, decreased immature form, and higher GAA enzymatic activity level. Similarly, in patient 16 , the post-treat- ment biopsy was morphologically worse than the pre- treatment one and we observed increase in both the mature and the immature GAA forms, with accumula- tions of both LC3II and P62. Despite these data, this patient showed the highest level of enzymatic activity.

In summary, our data showed that ERT positively modifies skeletal muscle pathology, at least in modestly to moderately affected LOPD patients. These results are clearer in the earlier phases of ERT administration, with a variable stability in the following years. Muscle biopsy improvement was evaluated in terms of reduced PAS-staining and disappearance of less severe vacuoles in posttreatment biopsies, both of which suggest a direct effect on reduction in the lysosomal glycogen pool. The evidence of treatment efficacy is further supported by biochemical findings showing mildly increased GAA enzymatic activity in all posttreatment muscle biopsies. Moreover, in 14 patients, western blot analysis demonstrated the conversion of the $110-\mathrm{kDa}$ precursor to mature $76 / 70-\mathrm{kDa}$ aglucosidase, strongly suggesting that the enzyme is targeted to lysosomes, where this proteolytic processing occurs [38]. Finally, most of these patients showed post-ERT improvement of autophagic flux, which contributes to GAA process- ing and maturation. Overall, we assessed the clinical, morphological and biochemical effects of ERT on skele- tal muscle tissue in a group of LOPD patients.

\section{Acknowledgements}

The authors thank Sanofi Genzyme for kindly providing the GAA Antibody, the Italian Association of Myology, the Associazione Amici del 'Centro Dino Ferrari', University of Milan, The Biobank of Skeletal Muscle, Peripheral Nerve, DNA and Cell Cultures, member of the Telethon Network of Genetic Biobanks (Project GTB 12001), funded by Telethon Italy, the Eurobiobank Network and Telethon Foundation for the Grant GUP13013 (A. Toscano).

\section{Author contributions}

MR, RV, MM, LM, MSc, AT, GPC and MM contributed to the study design. MR, RV, DR, VL, SS, IC, GF, AB,
FF and GPC contributed to the data collection. MR, RV, SM, MSa, AT, MSc and MM drafted the manuscript. OM, SP, MF, PT, MM, LM, TM and AT provided the clinical information and muscle biopsy specimens. AN, MSa and CA studied the autophagic function. SM per- formed the statistical analysis. All authors read and approved the final manuscript.

\section{Disclosures}

In the last 3 years, AT has received from Sanofi Gen- zyme some reimbursement for talking in teaching courses and because he also is member of Global Pompe Registry committee. MM and MSc received reimbursement for participation in board meetings and invited lectures by Sanofi Genzyme. Other Authors report no disclosures.

\section{References}

1 Raben N, Plotz P, Byrne BJ. Acid alpha-glucosidase deficiency (glycogenosis type II, Pompe disease). Curr Mol Med 2002; 2: 145-66 (Review)

2 Hagemans MLC, Winkel LPF, Van Doorn PA, Hop WJ, Loonen MC, Reuser AJ, Van der Ploeg AT. Clinical manifestations and natural course of lateonset Pom- pe's disease in 54 Dutch patients. Brain 2005; 128(Pt 3): 671-7

3 Angelini C, Semplicini C, Ravaglia S, Bembi B, Servidei S, Pegoraro E, Moggio M, Filosto M, Sette E, Cresci- manno G, Tonin P, Parini R, Morandi L, Marrosu G, Greco G, Musumeci O, Di IG, Siciliano G, Donati MA, Carubbi F, Ermani M, Mongini T, Toscano A; Italian GSDII Group. Observational clinical study in juvenile- adult glycogenosis type 2 patients undergoing enzyme replacement therapy for up to 4 years. $J$ Neurol 2012; 259: 952-8

4 Montagnese F, Granata F, Musumeci O, Rodolico C, Mondello S, Barca E, Cucinotta M, Ciranni A, Longo $\mathrm{M}$, Toscano A. Intracranial arterial abnormalities in patients with late onset Pompe disease (LOPD). J Inherit Metab Dis 2016; 39:391-8

5 Kikuchi T, Yang HW, Pennybacker M, Ichihara N, Mizutani M, Van Hove JL, Chen YT. Clinical and meta- bolic correction of Pompe disease by enzyme therapy in acid maltase-deficient quail. J Clin Invest 1998; 101: 827-33

6 Bijvoet AG, Van Hirtum H, Kroos MA, Van de Kamp EH, Schoneveld O, Visser P, Brakenhoff JP, Weggeman M, van Corven EJ, Van der Ploeg AT, Reuser AJ. Human acid alpha-glucosidase from rabbit milk has therapeutic effect in mice with glycogen storage disease type II. Hum Mol Genet 1999; 8: 2145-53 
7 Raben N, Danon M, Gilbert AL, Dwivedi S, Collins B, Thurberg BL, Mattaliano RJ, Nagaraju K, Plotz PH. Enzyme replacement therapy in the mouse model of Pompe disease. Mol Genet Metab 2003; 80: 159-69

8 Kishnani PS, Corzo D, Nicolino M, Byrne B, Mandel $\mathrm{H}$, Hwu WL, Leslie N, Levine J, Spencer C, McDonald M, Li J, Dumontier J, Halberthal M, Chien YH, Hopkin R, Vijayaraghavan S, Gruskin D, Bartholomew D, van der Ploeg A, Clancy JP, Parini R, Morin G, Beck M, De la Gastine GS, Jokic M, Thurberg B, Richards S, Bali D, Davison M, Worden MA, Chen YT, Wraith JE. Recom- binant human acid a-glucosidase. Major clinical bene- fits in infantile-onset Pompe disease. Neurology 2007; 68: 99-109.

9 Kishnani PS, Corzo D, Leslie ND, Gruskin D, Van der Ploeg A, Clancy JP, Parini R, Morin G, Beck M, Bauer MS, Jokic M, Tsai CE, Tsai BW, Morgan C, O'Meara T, Richards S, Tsao EC, Mandel H. Early treatment with alglucosidase alpha prolongs long-term survival of infants with Pompe disease. Pediatr Res 2009; 66: 329-35

10 Anderson LJ, Henley W, Wyatt KM, Nikolaou V, Waldek S, Hughes DA, Lachmann RH, Logan S. Effectiveness of enzyme replacement therapy in adults with late-onset Pompe disease: results from the NCS-LSD cohort study. J Inherit Metab Dis 2014; 37: $945-52$

11 Toscano A, Schoser B. Enzyme replacement therapy in late-onset Pompe disease: a systematic literature review. J Neurol 2013; 260: 951-9

12 Schoser B, Stewart A, Kanters S, Hamed A, Jansen J, Chan K, Karamouzian M, Toscano A. Survival and long-term outcomes in late-onset Pompe disease following alglucosidase alfa treatment: a systematic review and meta-analysis. J Neurol 2016; 264: $621-$ 30 (Review)

13 Feeney EJ, Austin S, Chien YH, Mandel H, Schoser B, Prater S, Hwu WL, Ralston E, Kishnani PS, Raben N. The value of muscle biopsies in Pompe disease: identi- fying lipofuscin inclusions in juvenile- and adult-onset patients. Acta Neuropathol Commun 2014; 2: 2

14 van der Ploeg A, Carlier PG, Carlier RY, Kissel JT, Schoser B, Wenninger S, Pestronk A, Barohn RJ, Dimachkie MM, Goker-Alpan O, Mozaffar T, Pena LD, Simmons Z, Straub V, Guglieri M, Young P, Boentert M, Baudin PY, Wens S, Shafi R, Bjartmar C, Thurberg BL. Prospective exploratory muscle biopsy, imaging, and functional assessment in patients with late-onset Pompe disease treated with alglucosidase alfa: the EMBASSY Study. Mol Genet Metab 2016; 119: 115-23

15 Nascimbeni AC, Fanin M, Masiero E, Angelini C, San- dri M. Impaired autophagy contributes to muscle atro- phy in glycogen storage disease type II patients. Autophagy 2012; 11: 1697-700

16 Schfnzer A, Kaiser AK, MGhlfeld C, Kulessa M, Paulus W, von Pein $\mathrm{H}$, Rohrbach M, Viergutz L, Mengel E,
Marquardt T, Neubauer B, Acker T, Hahn A. Quantifi- cation of muscle pathology in infantile Pompe disease. Neuromuscul Disord 2017; 27: 14152

17 Raben N, Baum R, Schreiner C, Takikita S, Mizushima N, Ralston E, Plotz P. When more is less: excess and deficiency of autophagy coexist in skeletal muscle in Pompe disease. Autophagy 2009; 5: $111-13$

18 Sandri M, Coletto L, Grumati P, Bonaldo P. Misregula- tion of autophagy and protein degradation systems in myopathies and muscular dystrophies. $J$ Cell Sci 2013; 126(Pt 23): 5325-33 (Review)

19 Nascimbeni AC, Fanin M, Masiero E, Angelini C, Sandri $\mathrm{M}$. The role of autophagy in the pathogenesis of glycogen storage disease type II (GSDII). Cell Death Dif-fer 2012; 19: 1698-708

20 Angelini C, Semplicini C, Ravaglia S, Moggio M, Comi GP, Musumeci O, Pegoraro E, Tonin P, Filosto M, Ser- videi S, Morandi L, Crescimanno G, Marrosu G, Sicil- iano G, Mongini T, Toscano A; Italian Group on GSDII. New motor outcome function measures in eval- uation of late-onset Pompe disease before and after enzyme replacement therapy. Muscle Nerve 2012; 45: 831-4

21 Nascimbeni AC, Fanin M, Tasca E, Angelini C. Molecu- lar pathology and enzyme processing in various phe- notypes of acid maltase deficiency. Neurology 2008; 70: 617-26

22 Regnery C, Kornblum C, Hanisch F, Vielhaber S, Strigl- Pill N, Grunert B, MGller-Felber W, Glocker FX, Spran- ger M, Deschauer M, Mengel E, Schoser B. 36 months observational clinical study of 38 adult Pompe patients under alglucosidase alfa enzyme replacement therapy. J Inherit Metab Dis 2012; 35: 837-45

23 Ripolone M, Ronchi D, Violano R, Vallejo D, Fagiolari G, Barca E, Lucchini V, Colombo I, Villa L, Berardinelli A, Balottin U, Morandi L, Mora M, Bordoni A, Fortunato F, Corti S, Parisi D, Toscano A, Sciacco M, DiMauro S, Comi GP, Moggio M. Impaired mus- cle mitochondrial biogenesis and myogenesis in spinal muscular atrophy. JAMA Neurol 2015; 72: 666-75

24 Peverelli L, Testolin S, Villa L, D'Amico A, Petrini S, Favero C, Magri F, Morandi L, Mora M, Mongini T, Bertini E, Sciacco M, Comi GP, Moggio M. Histologic muscular history in steroid-treated and untreated patients with Duchenne dystrophy. Neurology 2015; 85: $1886-93$

25 Kavsak PA, Ko DT, Wang X, Macrae AR, Jaffe AS. 2007 universal myocardial infarction definition change criteria for risk stratification by use of a highsensitivity cardiac troponin I assay. Clin Chem 2010; 56: 487-9

26 Apple FS, Jesse RL, Newby LK, Wu AH, Christenson RH. National Academy of Clinical Biochemistry and IFCC Committee for Standardization of Markers of Car- diac Damage Laboratory Medicine Practice Guidelines: 
analytical issues for biochemical markers of acute coronary syndromes. Circulation 2007; 115: e352-5

27 Gabellini D, D’Antona G, Moggio M, Prelle A, Zecca C, Adami R, Angeletti B, Ciscato P, Pellegrino MA, Bot- tinelli R, Green MR, Tupler R. Facioscapulohumeral muscular dystrophy in mice overexpressing FRG1. Nature 2006; 439: 973-7

28 Ausems MG, Lochman P, van Diggelen OP, Ploos vAH, Reuser AJ, Wokke JH. A diagnostic protocol for adult-onset glycogen storage disease type II. Neurology 1999;52:851-3.

29 Sandri M, Sandri C, Gilbert A, Skurk C, Calabria E, Picard A, Walsh K, Schiaffino S, Lecker SH, Goldberg AL. Foxo transcription factors induce the atrophy- related ubiquitin ligase atrogin-1 and cause skeletal muscle atrophy. Cell 2004; 117: 399-412

30 Raben N, Fukuda T, Gilbert AL, de Jong D, Thurberg BL, Mattaliano RJ, Meikle P, Hopwood JJ, Nagashima K, Nagaraju K, Plotz PH. Replacing acid alpha-glucosi- dase in Pompe disease: recombinant and transgenic enzymes are equipotent, but neither completely clears glycogen from type II muscle fibers. Mol Ther 2005; 11: 48-56

31 Hawes ML, Kennedy W, O'Callaghan MW, Thurberg BL. Differential muscular glycogen clearance after enzyme replacement therapy in a mouse model of Pompe disease. Mol Genet Metab 2007; 91: 343-51

32 Raben N, Ralston E, Chien YH, Baum R, Schreiner C, Hwu WL, Zaal KJ, Plotz PH. Differences in the predom- inanceof lysosomal and autophagic pathologies between infants and adults with Pompe disease: impli- cations for therapy. Mol Genet Metab 2010; 101: 324- 31

33 van den Berg LE, Drost MR, Schaart G, de Laat J, van Doorn PA, van der Ploeg AT, Reuser AJ. Muscle fiber- type distribution, fiber-type-specific damage, and the Pompe disease phenotype. J Inherit Metab Dis 2013; 36: 787-94

34 Schoser BG, M6ller-H6cker J, Horvath R, Gempel K, Pongratz D, Lochmiller H, MGller-Felber W. Adult- onset glycogen storage disease type 2: clinicopatholo- gical phenotype revisited. Neuropathol Appl Neurobiol 2007; 33: 544-59

35 Herzog A, Hartung R, Reuser AJ, Hermanns P, Runz H, Karabul N, G6kce S, Pohlenz J, Kampmann C, Lampe C, Beck M, Mengel E. A cross-sectional singlecentre study on the spectrum of Pompe disease, German patients: molecular analysis of the GAA gene, manifestation and genotype-phenotype correlations. Orphanet J Rare Dis 2012; 7: 35

36 van der Ploeg AT, Reuser AJ. Pompe's disease.

Lancet

2008; 372: 1342-53

37 van der Ploeg AT, Kruijshaar ME, Toscano A, Laforet P, Angelini C, Lachmann RH, Pascual SI, Roberts M, R6sler K, Stulnig T, van Doorn PA, Van den Bergh
PYK, Vissing J, Schoser B; European Pompe Consor- tium. European consensus for starting and stopping enzyme replacement therapy in adult patients with Pompe disease: a 10-year experience. Eur J Neurol 2017; 24: 768-e31

38 Schoser B, Hill V, Raben N. Therapeutic approaches in glycogen storage disease type II/Pompe disease. Neu-rotherapeutics 2008; 5: 569-78 (Review)

\section{Supporting information}

Additional Supporting Information may be found in the online version of this article at the publisher's website:

Figure S1. (A) Muscle biopsy evaluation. Two muscle biopsies, before and after ERT, from 18 patients were analysed. Vacuolated fibres were classified according to a four-point graded scale considering both the number and the size of vacuoles. Original magnification: 4009.

+ , normal or mild, fibres containing few and small vac- uoles; ++, moderate, fibres with more numerous small vacuoles and few large vacuoles; +++, severe, fibres with large vacuoles; ++++ , very severe, fibres with large vac- uoles that replaced most of the sarcoplasm. Mean values were obtained by each operator (three different opera- tors) for each biopsy and the final value, expressed in percentage, represents the mean of each operator mean value. (B) Vacuolation weighted delta (VWD) score chart for the assessment of response to the ERT in patients with Pompe disease according to their vacuolated fibres characteristics. Vacuolated fibres were classified accord- ing to a four-point graded scale considering both the number and the size of vacuoles. Pre- and post-ERT biop- sies were scored according to these criteria and a relative change $\geq 20 \%$ between pre- and post-ERT biopsies served as the criterion standard to determine relevant improve- ment or worsening. Vacuolation reduction $\geq 20 \%$ (opti- mal treatment response) was assigned a score of 0 point. The lack of changes in vacuolation (suboptimal treat- ment response) was given 0.5 point. Lastly, in line with a more severe degree of impairment of muscle fibres a dif- ferent weight was assigned to vacuolation increase

$\geq 20 \%$ depending on the different vacuolated fibres char- acteristics, with a minimum score of 1 to a maximum of

2. The VWD scores range from 1 to 8 , with scores of $\leq 4$ indicating an overall improvement/unmodified condi- tion, and $>4$ indicating an overall worsening. ${ }^{a}$ We chose to add plus 1 for simple mathematical reasons. 
Table S1. Clinical features of the 18 patients with late onset Pompe disease.

Table S2. PAS staining evaluation in each biopsy. Table S3. Histopathological features in pre- and post- ERT muscle biopsies of the 18 patients with late onset Pompe disease.
Table S4. Percentage of vacuolated fibres (type 1 or type 2) in each biopsy. 